\title{
Passivity-Based Analysis of Biochemical Networks Displaying Homeostasis
}

\author{
Daniel M. Tveit Kristian Thorsen \\ Department of Electrical Engineering and Computer Science, University of Stavanger, Norway, \\ \{daniel.m.tveit, kristian.thorsen\}@uis.no
}

\begin{abstract}
Homeostasis refers to the ability of organisms and cells to maintain a stable internal environment even in the presence of a changing external environment. On the cellular level many compounds such as ions, $\mathrm{pH}$, proteins, and transcription factors have been shown to be tightly regulated, and mathematical models of biochemical networks play a major role in elucidating the mechanisms behind this behaviour. Of particular interest is the control theoretic properties of these models, e.g. stability and robustness. The simplest models consist of two components, a controlled compound and a controller compound. We have previously explored how signalling between these two compounds can be arranged in order for the network to display homeostasis, and have constructed a class of eight two-component reaction kinetic networks with negative feedback that shows set-point tracking and disturbance rejection properties. Here, we take a closer look at the stability and robust control inherent to this class of systems. We show how these systems can be described as negative feedback connections of two nonlinear sub-systems, and show that both sub-systems are output strictly passive and zero-state detectable. Using a passivity-based approach, we show that all eight systems in this class of two-component networks are asymptotically stable.
\end{abstract}

Keywords: Passivity, homeostasis, adaptation, stability, robust control, integral control, negative feedback

\section{Introduction}

Control theoretic methods are useful when uncovering the mechanism behind cellular control processes. Especially properties such as stability and robust control are of interest. One structure with these properties is the negative feedback connection, which is employed in several biochemical processes, such as the regulation of enzyme synthesis and activity (Keener and Sneyd, 2009; Tyson and Othmer, 1978). Other more complicated control systems, such as the control of calcium in yeast cells, have also been analysed in this manner (Liu, 2012). We have previously investigated a class of simple two-component biochemical networks displaying homeostasis. These networks have been used in modelling ionic homeostasis in enterocytes (Thorsen et al., 2014), the development of biochemical controllers with robust control to perturbations changing rapidly in time (Fjeld et al., 2017), and developing yeast cells with an increased tolerance to high copper concentrations (Thorsen et al., 2016a). The networks consist of a controlled compound $x_{1}$ and a controller compound $x_{2}$, and through certain signalling reactions between these two compounds, the systems form negative feedback connections with integral action, giving robust control of $x_{1}$. We have identified eight such two-component systems, termed controller motifs (Drengstig et al., 2012). Figure 1 shows the structure of these controller motifs. For the class of two-component systems considered here, $x_{1}$ acts on either the synthesis or the degradation of $x_{2}$, and $x_{2}$ acts on either the synthesis or the degradation of $x_{1}$. This gives eight controller motifs with two components, each acting on the other through a single signalling reaction.

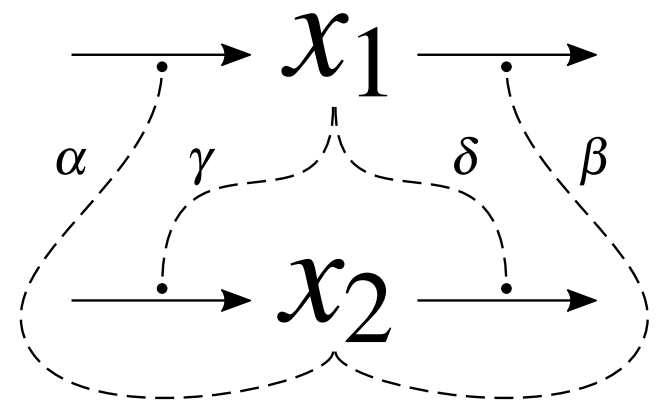

Figure 1. The controller motifs are formed by two compounds, $x_{1}$ and $x_{2}$, with signalling reactions $\alpha, \beta, \gamma, \delta$ between them. For each controller motif, there is one signalling reaction from $x_{1}$ acting on $x_{2}$, and one signalling reaction from $x_{2}$ acting on $x_{1}$. These signalling reactions form a negative feedback connection with the two compounds. There are in total eight such controller motifs.

In general, the controller motifs take the form

$$
\begin{aligned}
& \dot{x}_{1}=k_{s, 1} \cdot \alpha\left(x_{2}\right)-f_{d, 1}\left(x_{1}\right) \cdot \beta\left(x_{2}\right) \\
& \dot{x}_{2}=k_{s, 2} \cdot \gamma\left(x_{1}\right)-f_{d, 2}\left(x_{2}\right) \cdot \delta\left(x_{1}\right)
\end{aligned}
$$

where $k_{s, i}$ are positive rate constants determining the basal synthesis of $x_{i}$, the functions $f_{d, i}$ determine the degradation rate of $x_{i}$ following Michaelis-Menten kinetics (Cornish-Bowden, 2012), and $\alpha, \beta, \gamma, \delta$ are signalling functions between the two chemical compounds. Only one of the signalling functions $\alpha$ and $\beta$, and one of $\gamma$ and $\delta$, will be non-constant for a given controller motif. 
The Michaelis-Menten equation models the reaction rate of a compound $x_{i}$ by an enzyme reaction

$$
f_{d, i}\left(x_{i}\right)=\frac{k_{d, i} \cdot x_{i}}{K_{M, i}+x_{i}}
$$

where $f_{d, i}$ is the reaction rate, $k_{d, i}$ is a positive constant determining the maximal reaction rate, and $K_{M, i}$ is a positive constant called the Michaelis constant (Cornish-Bowden, 2012).

The signalling functions $\alpha, \beta, \gamma, \delta$ can either be activating or inhibiting. Activating signalling follow the expression for mixed activation (Cornish-Bowden, 2012)

$$
f_{a c t}\left(x_{i}\right)=\frac{x_{i}}{K_{A, i}+x_{i}}
$$

where the activation of some reaction is determined by the level of $x_{i}$, and $K_{A, i}$ is a positive constant for the activation reaction. Inhibiting signalling follow the expression for mixed inhibition (Cornish-Bowden, 2012)

$$
f_{\text {inh }}\left(x_{i}\right)=\frac{K_{I, i}}{K_{I, i}+x_{i}}
$$

where the amount of inhibition is determined by the level of $x_{i}$, and $K_{I, i}$ is a positive constant for the inhibition reaction.

\section{Stability}

To show asymptotic stability of the controller motifs, we first perform a change of variables, $z_{1}=x_{1}-x_{1}^{*}$ and $z_{2}=x_{2}-x_{2}^{*}$, where $\left(x_{1}^{*}, x_{2}^{*}\right)$ is the equilibrium point of the system. The states $x_{1}$ and $x_{2}$ represent physical concentrations of compounds. Therefore, a global result corresponds to positive values of the states and the equilibrium point. The change of variables moves the equilibrium point to the origin. Using the fact that $k_{s, 1} \cdot \alpha\left(x_{2}^{*}\right)=$ $f_{d, 1}\left(x_{1}^{*}\right) \cdot \beta\left(x_{2}^{*}\right)$ and $k_{s, 2} \cdot \gamma\left(x_{1}^{*}\right)=f_{d, 2}\left(x_{2}^{*}\right) \cdot \delta\left(x_{1}^{*}\right)$, the system is rewritten to the form

$$
\begin{aligned}
& \dot{z}_{1}=-f_{1}\left(z_{1}\right)+h_{2, \alpha}\left(z_{2}\right)-g_{1}\left(z_{1}\right) \cdot h_{2, \beta}\left(z_{2}\right) \\
& \dot{z}_{2}=-f_{2}\left(z_{2}\right)+h_{1, \gamma}\left(z_{1}\right)-g_{2}\left(z_{2}\right) \cdot h_{1, \delta}\left(z_{1}\right)
\end{aligned}
$$

where these new functions are defined as follows

$$
\begin{aligned}
f_{1}\left(z_{1}\right) & =f_{d, 1}\left(z_{1}+x_{1}^{*}\right) \cdot \beta\left(x_{2}^{*}\right)-f_{d, 1}\left(x_{1}^{*}\right) \cdot \beta\left(x_{2}^{*}\right) \\
f_{2}\left(z_{2}\right) & =f_{d, 2}\left(z_{2}+x_{2}^{*}\right) \cdot \delta\left(x_{1}^{*}\right)-f_{d, 2}\left(x_{2}^{*}\right) \cdot \delta\left(x_{1}^{*}\right) \\
g_{1}\left(z_{1}\right) & =f_{d, 1}\left(z_{1}+x_{1}^{*}\right) \\
g_{2}\left(z_{2}\right) & =f_{d, 2}\left(z_{2}+x_{2}^{*}\right) \\
h_{1, \gamma}\left(z_{1}\right) & =k_{s, 2} \cdot \gamma\left(z_{1}+x_{1}^{*}\right)-k_{s, 2} \cdot \gamma\left(x_{1}^{*}\right) \\
h_{1, \delta}\left(z_{1}\right) & =\delta\left(z_{1}+x_{1}^{*}\right)-\delta\left(x_{1}^{*}\right) \\
h_{2, \alpha}\left(z_{2}\right) & =k_{s, 1} \cdot \alpha\left(z_{2}+x_{2}^{*}\right)-k_{s, 1} \cdot \alpha\left(x_{2}^{*}\right) \\
h_{2, \beta}\left(z_{2}\right) & =\beta\left(z_{2}+x_{2}^{*}\right)-\beta\left(x_{2}^{*}\right)
\end{aligned}
$$

The functions $f_{i}$ and $h_{i}$ are strictly increasing and satisfy $f_{i}(0)=h_{i}(0)=0$ for the interval $\left(-x_{i}^{*}, \infty\right)$. The functions $g_{i}>0$ for the same interval. As noted earlier, only one of the signalling functions $\alpha$ and $\beta$, and one of $\gamma$ and $\delta$ will be non-constant for a given controller motif. This means that only one of the output functions $h_{2, \alpha}$ and $h_{2, \beta}$, and one of $h_{1, \gamma}$ and $h_{1, \delta}$ will be non-zero. In addition, if the signalling functions $\alpha, \beta, \gamma, \delta$ are inhibiting instead of activating, the corresponding output functions $h_{i}$ are defined to be negative. For example, the controller motif shown in Figure 2 has non-constant signalling functions $\beta$ and $\delta$, while $\alpha=\gamma=1$. Therefore, the corresponding output functions $h_{2, \beta}$ and $h_{1, \delta}$ are non-zero, while $h_{2, \alpha}=h_{1, \gamma}=0$. In addition, the signalling function $\delta$ is inhibiting, and the corresponding output function $h_{1, \delta}$ is defined to be negative.

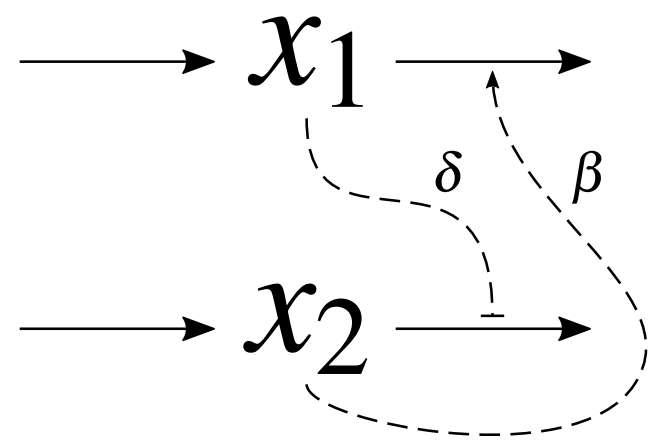

Figure 2. One of eight controller motifs that form a negative feedback connection. In this case, $x_{1}$ is acting on $x_{2}$ by inhibiting its degradation, and $x_{2}$ is acting on $x_{1}$ by activating its degradation.

The system equations for this controller motif are given by

$$
\begin{aligned}
& \dot{x}_{1}=k_{s, 1}-f_{d, 1}\left(x_{1}\right) \cdot \beta\left(x_{2}\right)=k_{s, 1}-\frac{k_{d, 1} \cdot x_{1}}{K_{M, 1}+x_{1}} \cdot \frac{x_{2}}{K_{A, 2}+x_{2}} \\
& \dot{x}_{2}=k_{s, 2}-f_{d, 2}\left(x_{2}\right) \cdot \delta\left(x_{1}\right)=k_{s, 2}-\frac{k_{d, 2} \cdot x_{2}}{K_{M, 2}+x_{2}} \cdot \frac{K_{I, 1}}{K_{I, 1}+x_{1}}
\end{aligned}
$$

and the transformed system equations are then

$$
\begin{aligned}
& \dot{z}_{1}=-f_{1}\left(z_{1}\right)-g_{1}\left(z_{1}\right) \cdot h_{2}\left(z_{2}\right) \\
& \dot{z}_{2}=-f_{2}\left(z_{2}\right)+g_{2}\left(z_{2}\right) \cdot h_{1}\left(z_{1}\right)
\end{aligned}
$$

where $h_{1}=h_{1, \delta}$ and $h_{2}=h_{2, \beta}$.

To show that the controller motifs are asymptotically stable, we think of them as negative feedback connections of two sub-systems. In general, these sub-systems take the form

$$
\begin{gathered}
H_{1}:\left\{\begin{array}{l}
\dot{z}_{1}=-f_{1}\left(z_{1}\right)+g_{1}\left(z_{1}\right) \cdot u_{1} \\
y_{1}=h_{1}\left(z_{1}\right)
\end{array}\right. \\
H_{2}:\left\{\begin{array}{l}
\dot{z_{2}}=-f_{2}\left(z_{2}\right)+g_{2}\left(z_{2}\right) \cdot u_{2} \\
y_{2}=h_{2}\left(z_{2}\right)
\end{array}\right.
\end{gathered}
$$


where the negative feedback connection can be formed either by having

$$
u_{1}=-y_{2}, \quad u_{2}=y_{1}
$$

or by

$$
u_{1}=y_{2}, \quad u_{2}=-y_{1}
$$

This corresponds to which of $H_{1}$ and $H_{2}$ is in the negative feedback.

\subsection{Passivity}

The next step is to determine if the two sub-systems are output strictly passive. This is done by using the storage function $S_{i}$ for sub-system $H_{i}$

$$
S_{i}=\int_{0}^{z_{i}} \frac{h_{i}(\sigma)}{g_{i}(\sigma)} \mathrm{d} \sigma
$$

where $h_{i}$ and $g_{i}$ are the functions in (20) and (21). The derivative of $S$ along trajectories is then

$$
\dot{S}_{i}=\frac{h_{i}\left(z_{i}\right)}{g_{i}\left(z_{i}\right)} \cdot \dot{z}_{i}=-f_{i}\left(z_{i}\right) \cdot \frac{h_{i}\left(z_{i}\right)}{g_{i}\left(z_{i}\right)}+u_{i} \cdot y_{i}
$$

The sub-systems are output strictly passive if the following inequality is satisfied (Khalil, 2002; Sepulchre et al., 1997)

$$
\dot{S}_{i} \leq-y_{i} \cdot \rho_{i}\left(y_{i}\right)+u_{i} \cdot y_{i}
$$

where $y_{i} \cdot \rho_{i}\left(y_{i}\right)>0 \forall y_{i} \neq 0$. Systems whose stored energy can only increase through the supply of an external source, are passive (Khalil, 2002). For inequality (26), the "energy" absorbed by the system, $u_{i} \cdot y_{i}$, is greater than the increase in stored "energy", $\dot{S}_{i}$. In addition, the system has an "excess" of passivity from the term $y_{i} \cdot \rho\left(y_{i}\right)$. For the controller motifs, we choose $y_{i} \cdot \rho_{i}\left(y_{i}\right)=p_{i} \cdot y_{i}^{2}$, where $p_{i}$ is a positive constant. Inequality (26) is then satisfied by choosing $p_{i}$ such that

$$
0<p_{i} \leq \frac{f_{i}\left(z_{i}\right)}{h_{i}\left(z_{i}\right) \cdot g_{i}\left(z_{i}\right)}
$$

for the interval $\left(-x_{i}^{*}, \infty\right)$. For the same interval, the righthand side expression can be shown to be greater than zero, and either strictly increasing, or strictly decreasing. Inequality (27) is then satisfied by finding $p_{i}$ as the lower bound of the right-hand side expression. The lower bound is given by the minimum value of the right-hand side expression at the limits $z_{i} \rightarrow-x_{i}^{*}$ and $z_{i} \rightarrow \infty$. Therefore, the value of $p_{i}$ is determined by

$$
p_{i}=\min \left\{\lim _{z_{i} \rightarrow-x_{i}^{*+}} \frac{f_{i}\left(z_{i}\right)}{h_{i}\left(z_{i}\right) \cdot g_{i}\left(z_{i}\right)}, \lim _{z_{i} \rightarrow \infty} \frac{f_{i}\left(z_{i}\right)}{h_{i}\left(z_{i}\right) \cdot g_{i}\left(z_{i}\right)}\right\}
$$

Thus, the sub-systems (20) and (21) are output strictly passive.
It has been shown that the negative feedback connection of two output strictly passive systems is asymptotically stable if the sub-systems are zero-state detectable (Sepulchre et al., 1997). To show that $H_{1}$ and $H_{2}$ are zerostate detectable, we consider the system

$$
H:\left\{\begin{array}{l}
\dot{z}=f(z, u) \\
y=h(z, u)
\end{array}\right.
$$

with $u=0 . \quad H$ is said to be zero-state detectable if the origin is asymptotically stable conditionally to $Z$, where $Z$ is the largest positively invariant set in $\left\{z \in \mathbb{R}^{n} \mid y=h(z, 0)=0\right\}$. For the special case when $Z=$ $\{0\}$, we say that $H$ is zero-state observable (Khalil, 2002; Sepulchre et al., 1997). We now consider the sub-systems $H_{1}$ and $H_{2}$, with inputs $u_{1}=u_{2}=0$. From the the output functions (12)-(15), it can be seen that

$$
y_{1}=y_{2}=0 \Longrightarrow z_{1}=z_{2}=0
$$

Therefore, the sub-systems $H_{1}$ and $H_{2}$ are zero-state observable if the origin is locally asymptotically stable. We verify this by linearisation of the sub-systems at the origin. With $u_{i}=y_{i}=0$, the system equations of (20) and (21) are reduced to $\dot{z}_{i}=-f_{i}\left(z_{i}\right)$, and linearisation gives

$$
\begin{aligned}
& H_{1}:\left.\frac{\partial\left(-f_{1}\right)}{\partial z_{1}}\right|_{z_{1}=0}=-\frac{k_{d, 1} \cdot K_{M, 1}}{\left(K_{M, 1}+x_{1}^{*}\right)^{2}} \cdot \beta\left(x_{2}^{*}\right)<0 \\
& H_{2}:\left.\frac{\partial\left(-f_{2}\right)}{\partial z_{2}}\right|_{z_{2}=0}=-\frac{k_{d, 2} \cdot K_{M, 2}}{\left(K_{M, 2}+x_{2}^{*}\right)^{2}} \cdot \delta\left(x_{1}^{*}\right)<0
\end{aligned}
$$

where $f_{i}$ are the functions given by (8) and (9).

Finally, to show that the entire system is asymptotically stable, we use the combined storage function

$$
S=S_{1}+S_{2}
$$

Because $S_{1}$ and $S_{2}$ are positive definite, so is $S$. Since the two sub-systems form a negative feedback connection given by (22) or (23), the derivative of $S$ along trajectories is reduced to

$$
\dot{S}=\dot{S}_{1}+\dot{S}_{2} \leq-p_{1} \cdot y_{1}^{2}-p_{2} \cdot y_{2}^{2}
$$

which is negative definite. This shows that all bounded solutions converge to the set $\left\{\left(z_{1}, z_{2}\right) \mid y_{1}=y_{2}=0\right\}$. From (30), we know that this corresponds to the origin. Since the origin has been shown to be locally asymptotically stable by linearisation, we conclude that the controller motifs are asymptotically stable. If the storage functions $S_{1}$ and $S_{2}$ are radially unbounded, so is $S$, and the controller motifs are globally asymptotically stable.

\section{Integral Control}

The system given by equations (1) and (2) can be shown to include integral control. This is done by rewriting equation (2). For example, the controller motif given by equations (16) and (17), shown in Figure 2, can have equation 
(17) rewritten to

$$
\dot{x}_{2}=-\frac{k_{s, 2}}{K_{I, 1}+x_{1}} \cdot\left(\frac{x_{2}}{K_{M, 2}+x_{2}} \cdot \frac{k_{d, 2} \cdot K_{I, 1}}{k_{s, 2}}-K_{I, 1}-x_{1}\right)
$$

For $K_{M, 2}=0$, this is reduced to

$$
\begin{aligned}
\dot{x}_{2} & =-\frac{k_{s, 2}}{K_{I, 1}+x_{1}} \cdot\left(\frac{k_{d, 2} \cdot K_{I, 1}}{k_{s, 2}}-K_{I, 1}-x_{1}\right) \\
& =G_{i} \cdot\left(x_{1, \text { set }}-x_{1}\right)
\end{aligned}
$$

which has the form of the integral control law. This means that for $K_{M, 2}=0$, or $K_{M, 2} \ll x_{2}$, the system behaves as an integral controller for $x_{1}$ with a set-point given by

$$
x_{1, s e t}=\frac{k_{d, 2} \cdot K_{I, 1}}{k_{s, 2}}-K_{I, 1}
$$

For the case when $K_{M, 2}>0$, the set-point in (35) is defined as

$$
x_{1, \text { set }}=\frac{x_{2}}{K_{M, 2}+x_{2}} \cdot \frac{k_{d, 2} \cdot K_{I, 1}}{k_{s, 2}}-K_{I, 1}
$$

Thus, there is still integral action, however, the set-point changes with perturbations. The effect of this is shown in Figure 3, where the case with $K_{M, 2}=0$ results in perfect adaptation to perturbations in the synthesis of $x_{1}$, and the case with $K_{M, 2}>0$ results in only partial adaptation.

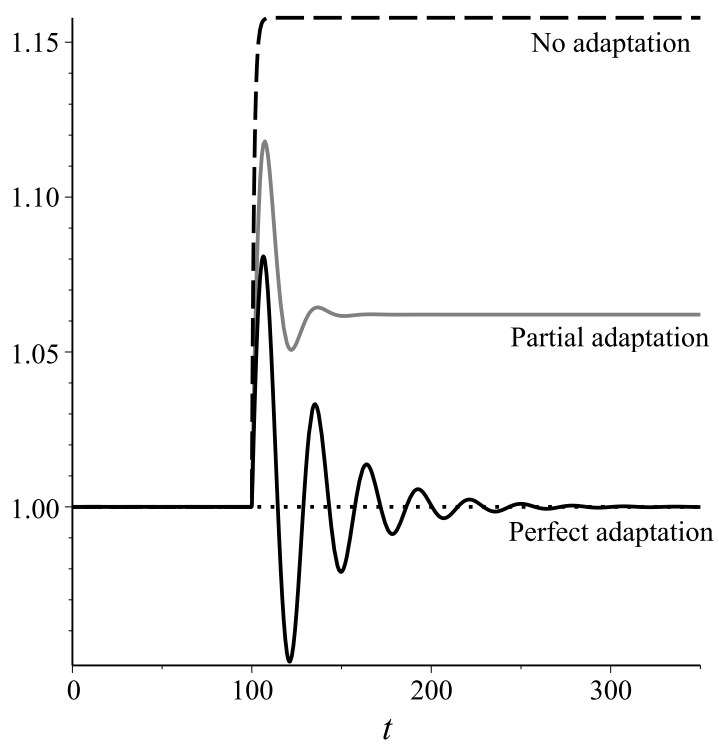

Figure 3. The response to a step-wise perturbation in the synthesis of $x_{1}$. Dashed line shows no adaptation, grey line shows partial adaptation, and black line shows perfect adaptation. These three cases correspond to the controller motifs having no integral control (no signalling between $x_{1}$ and $x_{2}$ ), integral control with $K_{M, 2}>0$, and integral control with $K_{M, 2}=0$, respectively.

It has previously been shown that the all the controller motifs include integral control (Drengstig et al., 2012;
Thorsen et al., 2016b). The system equation of the controller compound $x_{2}$ is written to the form of the integral control law

$$
\dot{x}_{2}=G_{i} \cdot\left(x_{1, \text { set }}-x_{1, \text { meas }}\right)
$$

Where $G_{i}$ is the controller gain, $x_{1, \text { set }}$ is the set point of the controlled compound $x_{1}$, and $x_{1 \text {,meas }}$ is a measurement function of $x_{1}$. Just like the system in Figure 2, the other controller motifs show partial or perfect adaptation depending on the value of $K_{M, 2}$ (Drengstig et al., 2012).

Because we are able to show that the controller motifs are asymptotically stable, as well as incorporating integral control, they must be robust to all parameter perturbations that do not destroy the stability of the closed-loop system (Khalil, 2002). An implication of asymptotic stability, is that the error $x_{1, \text { set }}-x_{1, \text { meas }}$ must be zero at the equilibrium point. With any parameter perturbation that does not destroy the stability of the closed-loop system, the equilibrium point may change, however, the error must return to zero. Thus, regulation will be achieved for as long as the perturbed equilibrium point remains asymptotically stable.

\section{Example}

We demonstrate our approach by considering the controller motif shown in Figure 2, given by the system equations (16) and (17). This system is transformed to the system equations given by (18) and (19). The transformed system can be represented as a negative feedback connection of two sub-systems $H_{1}$ and $H_{2}$, given by (20) and (21), with

$$
u_{1}=-y_{2}=-h_{2}\left(z_{2}\right), \quad u_{2}=y_{1}=h_{1}\left(z_{1}\right)
$$

We use some arbitrary values for the constants $k_{s, 1}=$ $1, k_{s, 2}=1, k_{d, 1}=3, k_{d, 2}=4, K_{M, 1}=1.5, K_{M, 2}=0.75$, $K_{I, 1}=1.5$, and $K_{A, 2}=2$, such that $x_{1}^{*}, x_{2}^{*}>0$. Thereby, the storage function for $H_{1}$ is given by

$$
S_{1}=\int_{0}^{z_{1}}\left(0.114-\frac{0.329}{\sigma+2.893}\right) \mathrm{d} \sigma
$$

and the derivative of $S_{1}$ along trajectories satisfy the inequality

$$
\dot{S}_{1} \leq-p_{1} \cdot y_{1}^{2}+u_{1} \cdot y_{1}
$$

with the constant $p_{1}$ determined by

$$
0<p_{1} \leq \frac{f_{1}\left(z_{1}\right)}{h_{1}\left(z_{1}\right) \cdot g_{1}\left(z_{1}\right)}=0.506+\frac{0.759}{z_{1}+2.893}
$$

For the interval $\left(-x_{1}^{*}, \infty\right)$, the right-hand side is always greater than or equal to 0.506 , and so we choose this value for $p_{1}$. This is illustrated in Figure 4. Similarly, for subsystem $\mathrm{H}_{2}$, the inequality

$$
0<p_{2} \leq \frac{f_{2}\left(z_{2}\right)}{h_{2}\left(z_{2}\right) \cdot g_{2}\left(z_{2}\right)}=0.185+\frac{0.370}{z_{2}+2.050}
$$




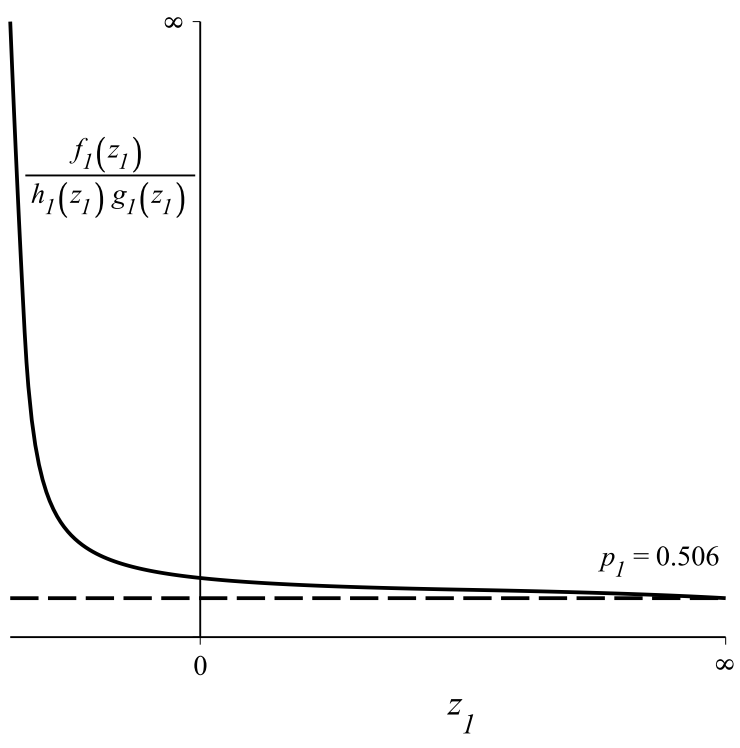

Figure 4. To determine the value of $p_{1}$ in inequality (44), we find the lower bound of the right-hand side expression. The figure shows that the lower bound is given by the limit of the righthand side expression as $z_{1} \rightarrow \infty$. In this case $p_{1}=0.506$.

is satisfied by choosing $p_{2}=0.185$, such that

$$
\dot{S}_{2} \leq-p_{2} \cdot y_{2}^{2}+u_{2} \cdot y_{2}
$$

where the storage function $S_{2}$ is given by

$$
S_{2}=\int_{0}^{z_{2}}\left(0.123-\frac{0.095}{\sigma+2.050}-\frac{0.312}{\sigma+4.050}\right) \mathrm{d} \sigma
$$

Thus, the combined storage function $S=S_{1}+S_{2}$ is positive definite and radially unbounded, because $S_{1}$ and $S_{2}$ are both positive definite and radially unbounded. In addition, the derivative of $S$ along trajectories satisfies

$$
\dot{S} \leq-0.506 \cdot y_{1}^{2}-0.185 \cdot y_{2}^{2}
$$

which implies that $\dot{S}$ is negative definite. $S$ and $\dot{S}$ are shown in Figure 5.
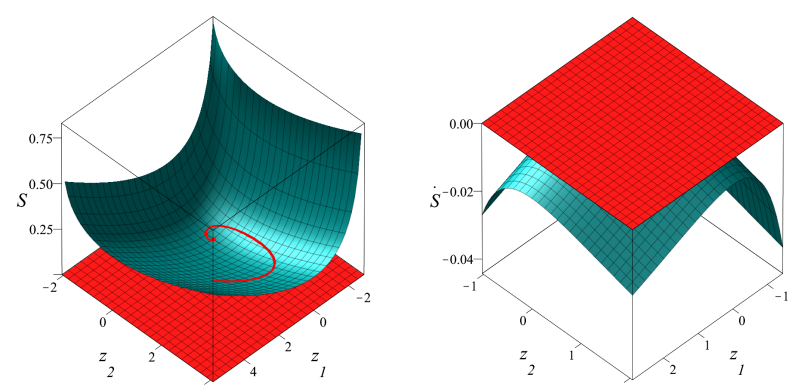

Figure 5. The storage function $S=S_{1}+S_{2}$ is shown to the left, and its derivative along trajectories is shown to the right. The red surfaces are at zero. We see that $S$ is positive definite, and $\dot{S}$ is negative definite. Therefore, all bounded solutions must converge to the set where $y_{1}=y_{2}=0$. A trajectory converging to the origin is shown as a red curve within the bowl formed by $S$ in the left figure.
Similarly to Lyapunov functions, we use the combined storage function $S$, and its derivative along trajectories $\dot{S}$ to draw conclusions about the stability of the system. The difference being that although the combined storage function is positive definite, and its derivative along trajectories negative definite, asymptotic stability is not implied. Instead it merely implies that all bounded solutions converge to the set where the outputs $y_{1}=y_{2}=0$. In general, this set could correspond to a number of values $\left(z_{1}, z_{2}\right)$, however, because the output functions (12)-(15) are strictly increasing and satisfy $h_{i}(0)=0$, this set corresponds to the origin. This implies that the sub-systems $H_{1}$ and $H_{2}$ are zero-state observable if the origin is locally asymptotically stable. This is shown by linearisation at the origin, using equations (31) and (32)

$$
\begin{aligned}
& H_{1}:\left.\frac{\partial\left(-f_{1}\right)}{\partial z_{1}}\right|_{z_{1}=0}=-0.118<0 \\
& H_{2}:\left.\frac{\partial\left(-f_{2}\right)}{\partial z_{2}}\right|_{z_{2}=0}=-0.131<0
\end{aligned}
$$

Thus, the sub-systems are zero-state observable, and the entire system must be asymptotically stable. In addition, as noted earlier, the combined storage function $S$ is radially unbounded, and therefore the system is globally asymptotically stable.

\section{Conclusion}

In this paper we have shown that a class of eight twocomponent biochemical networks displaying homeostasis, called controller motifs, are asymptotically stable. We have shown that the general system equations for these networks can be represented as negative feedback connections of two individual sub-systems. Then, these subsystems are shown to be output strictly passive, and the feedback connection in its entirety is shown to be asymptotically stable. In addition, it is shown that the controller motifs are robust to perturbations because they incorporate integral control.

When modelling cellular processes, it is beneficial to know that uncertainties in parameters do not fundamentally change the behaviour of the model. Because the controller motifs are asymptotically stable with integral action, processes which can be modelled within the framework of the controller motifs will have a qualitative behaviour which aligns well with experimental measurements, even with large uncertainties in parameter values. On the other hand, processes which do not conform well to the controller motifs can have wildly different qualitative responses in the face of parameter uncertainties, and the controller motifs can be excluded as models for such processes. Thus, it is not a matter of parameter tuning. This is a helpful property of the controller motifs, especially in system identification.

Here we have chosen to focus on controller motifs with zero-order synthesis, degradation by an enzyme reaction, and signalling following mixed activation/inhibition. 
However, other functions could be used. For example, we could use Hill kinetics for the degradation of the two compounds, or we could use linear activation for the signalling functions. In that case we would have to make sure that the properties assumed still hold.

\section{References}

Athel Cornish-Bowden. Fundamentals of Enzyme Kinetics. Wiley-Blackwell, fourth edition, 2012. ISBN 978-3-52733074-4.

T. Drengstig, I. W. Jolma, X. Y. Ni, K. Thorsen, X. M. Xu, and P. Ruoff. A basic set of homeostatic controller motifs. Biophysical Journal, 103(9):2000-2010, 2012. ISSN 00063495. doi:10.1016/j.bpj.2012.09.033.

Gunhild Fjeld, Kristian Thorsen, Tormod Drengstig, and Peter Ruoff. The Performance of Homeostatic Controller Motifs Dealing with Perturbations of Rapid Growth and Depletion. The Journal of Physical Chemistry B, 2017. ISSN 1520-6106. doi:10.1021/acs.jpcb.7b01989. URL http: //pubs . acs . org/doi/abs/10.1021/acs. jpcb.7b01989.

James Keener and James Sneyd. Mathematical Physiology I: Cellular Physiology. Springer, second edition, 2009. ISBN 978-0-387-75846-6. doi:10.1007/978-0-387-75847-3.

Hassan K. Khalil. Nonlinear Systems. Prentice Hall, third edition, 2002. ISBN 0-13-067389-7.

Weijiu Liu. Introduction to Modeling Biological Cellular Control Systems. Springer, 2012. ISBN 978-88-470-2489-2. doi:10.1007/978-88-470-2490-8.

Rodolphe Sepulchre, Mrdjan Jankovic, and Petar Kokotovic. Constructive Nonlinear Control. Springer, 1997. doi:10.1007/978-1-4471-0967-9.

K. Thorsen, T. Drengstig, and P. Ruoff. Transepithelial glucose transport and $\mathrm{Na}+/ \mathrm{K}+$ homeostasis in enterocytes: an integrative model. AJP: Cell Physiology, 307(4):C320-C337, 2014. ISSN 0363-6143. doi:10.1152/ajpcell.00068.2013. URL http: / / ajpcell.physiology.org/content / $307 / 4 / C 320$.

K. Thorsen, G. Risvoll, M. Mazumder, and D. R. McMillen. A controller for regulation of intracellular copper in S. cerevisiae. In Poster presented at ICSB2016, 2016a. doi:10.3252/pso.eu.17ICSB.2016. URL http: //www. postersessiononline.eu/pr/ congreso.asp?cod=392299830.

Kristian Thorsen, Geir B Risvoll, Daniel M Tveit, Peter Ruoff, and Tormod Drengstig. Tuning of Physiological Controller Motifs. In 2016 9th EUROSIM Congress on Modelling and Simulation, pages 28-33, 2016b. ISBN 9781509041190. doi:10.1109/EUROSIM.2016.174.

John J. Tyson and Hans G. Othmer. The Dynamics of Feedback Control Circuits in Biochemical Pathways. In Progress in Theoretical Biology Volume 5, pages 1-62. Academic Press, 1978. ISBN 0-12-543105-8. 